J. Clin. Chem. Clin. Biochem.

Vol. 18,1980 , pp. $867-877$

\title{
Klinisch-chemische Diagnostik depressiver Syndrome mit Hilfe eines neuroendokrinen Funktionstestes
}

\author{
Von K. Kleesiek
}

Abteilung Klinische Chemie und Pathobiochemie, Klinisch-chemisches Zentrallaboratorium (Vorstand: Prof. Dr. Dr. H. Greiling),

\section{A. Czernik}

\section{Abteilung Psychiatrie}

(Vorstand: Prof. Dr. W. Klages) und

\section{A. Eberhard}

\section{Abteilung Klinische Chemie und Pathobiochemie, Klinisch-chemisches Zentrallaboratorium (Vorstand: Prof. Dr. Dr. H. Greiling)}

der Rhein.-Westf. Technischen Hochschule Aachen

(Eingegangen am 24. Oktober 1979/19. Februar 1980)

Zusammenfassung: Bei 52 depressiv Erkrankten und 12 früher depressiv Erkrankten im psychosefreien Intervall wurde die Stimulierbarkeit der hypophysären Hormonsekretion mit Thyroliberin und unter Insulin-induzierter Hypoglykämie untersucht. Der Verlauf der Somatotropin-, Cortisol-, Prolactin-, Thyrotropin- und Glucosekonzentration im Serum wurde über 2 Stunden verfolgt. Es zeigen sich Unterschiede der neuroendokrinen Reaktion zwischen endogen und reaktiv (neurotisch) depressiv Erkrankten sowie Veränderungen während des Erkrankungsverlaufs:

1. Die Stimulierbarkeit der Somatotropin- und Cortisolsekretion und der Glucoseabfall ist bei endogen Depressiven gegenüber reaktiv Depressiven und gesunden Kontrollen vermindert.

2. Stimulationsmaxima der Somatotropinsekretion unter $40 \mathrm{mU} / 1$ bei depressiv Erkrankten während der depressiven Phase ermöglichen mit einer Vertrauenswahrscheinlichkeit von 0,9 eine Abgrenzung der endogenen Depression von der reaktiven Form.

3. Nach abgelaufener endogener Depression ist zwar eine Zunahme der Stimulierbarkeit der Somatotropin- und Cortisolsekretion nachweisbar, allerdings liegen auch zu diesem Zeitpunkt die Somatotropin-Maxima unterhalb von denen reaktiv Depressiver.

4. Bei Lithium-behandelten, psychosefreien Probanden ist die Stimulierbarkeit der Somatotropinsekretion nicht von der gesunder Kontrollen zu unterscheiden. Die Thyrotropinsekretion ist gesteigert. Im Vergleich mit gesunden Kontrollen ist auch bei diesen Probanden noch in einem hohen Prozentsatz (66\%)'eine abgeschwächte Insulininduzierte Hypoglykämie nachweisbar.

5. Angst und Agitiertheit haben während der depressiven Phase einen hemmenden Einfluß auf die Stimulierbarkeit der Prolactinsekretion.

Der hypophysäre Stimulationstest bietet die Möglichkeit, gestörtte neuroendokrine Funktionen bei depressiv Erkrankten zu erfassen und die Differentialdiagnose depressiver Syndrome zu objektivieren.

\section{Clinical chemical diagnosis of affective diseases by means of a neuroendocrine function test}

Summary: The stimulation of pituitary hormone secretion with thyroliberin and by insulin-induced hypoglycemia was investigated on 52 depressive patients and 12 postdepressive, lithiumtreated subjects. The serum concentrations of somatotropin, cortisol, prolactin, thyrotropin and glucose were followed for 2 hours. Differences in the neuroen- 
docrine response between endogenous and reactive (neurotic) depressive patients were observed, and changes during the course of the diseases were also recorded.

1. Stimulation of somatotropin and cortisol secretion, and the decrease of glucose are diminished in endogenous depressive patients, as compared to those of reactive depressive patients and healthy controls.

2. Somatotropin stimulation maxima below $40 \mathrm{mU} / 1$ in depressive patients during the depressive phase are indicative of endogenous depression, rather than the reactive form, with a confidence probability of 0.9 .

3. An increase in the stimulation of somatotropin and cortisol secretion can be shown after termination of endogenous depression. However, the somatotropin maxima are then also lower than those found in reactive depression.

4. The stimulation of somatotropin secretion in lithiumtreated psychosis-free subjects shows no difference from that of healthy controls; thyrotropin secretion increases. The weak, insulin-induced hypoglycemia, observed in these subjects, is higher $(66 \%)$ than in the healthy controls.

5. Fear and agitation have an inhibitory effect on the stimulation of the prolactin secretion during the depressive phase.

The pituitary stimulation test offers a means of ascertaining the disturbed neuroendocrine function in affective diseases and objectifying the differential diagnosis of depressive syndromes.

\section{Einführung}

An der Regulation der Hormonsekretion im hypothalamisch-hypophysären System sind noradrenerge, dopaminerge und serotonerge Neurone wesentlich beteiligt. Es besteht zudem eine enge morphologische und funktionelle Verknüpfung zwischen solchen Neuronen, die die Freisetzung von „Releasing-Hormonen" steuern, und Strukturen, die für das psychische Verhalten und Befinden sowie vegetative Funktionen von Bedeutung sind (1). Bisherige psychopharmakologische und neuroendokrinologische Befunde bei endogen Depressiven weisen auf einen Mangel catecholaminerger Amine im synaptischen Spalt und/oder eine Veränderung der Empfindlichkeit postsynaptischer Rezeptoren hin $(2,3)$.

Im Mittelpunkt neuroendokrinologischer Untersuchun: gen des depressiven Syndroms steht besonders das Hypothalamus-Hypophysen-Nebennierenrinden-System. Auf die Corticoliberin-Freisetzung haben nach heutigen Vorstellungen $\mathrm{GABA}^{-}$) und noradrenerge Neurone eine tonisch inhibitorische, serotonerge und cholinerge Neurone eine stimulatorische Wirkung $(4,5)$. Bei endogen Depressiven ist der hemmende Einfluß zentraler Neurone auf die Corticotropin-Freisetzung und damit die charakteristische Tagesrhythmik der Cortisolsekretion aufgehoben $(6,7,8)$. Es treten häufigere Cortisolsekretionsepisoden auf, die vor allem in den Nachmittags- und Nachtstunden zu einer höheren Cortisolkonzentration im Serum führen. Der Dexamethason-Hemmtest zeigt im Vergleich zu Gesunden und neurotisch Depressiven während der depressiven Phase eine geringere Hemmung der Corticotropin- und Cortisolsekretion $(9,10,11,12)$. Aứch im Insulin-Hypoglykämie-Test werden Funktionsstörungen in diesem System gefunden. So wird bei endogen Depressiven ein geringerer, bei reaktiv, neurotisch Depressiven ein verstärkter Sekretionsanstieg beschrieben (13).

Auch Veränderungen der Sómatotropinsekretion sind bei endogen Depressiven nachgewiesen worden. Die Somatotropinkonzentration, im Serum wird über Glucorezepto-

1) $\gamma$-Aminobuttersäure. ren, die wahrscheinlich im Nucleus ventromedialis lokalisiert sind, moduliert. Auf die verstärkte Somatotropinsekretion nach Insulin-induzierter Hypoglykämie haben noradrenerge Neurone einen fördernden Einfluß $(14,15)$. Außerdem scheinen serotonerge und dopaminerge Neurone eine fördernde Wirkung auf die Freisetzung eines Somatoliberins zu besitzen $(15,16,17)$. Gehemmt wird die Somatotropinsekretion durch Somatostatin, über dessen Beeinflussung durch aminerge Neurone wenig bekannt ist.

Bei endogener Depression ist die nach Insulin-induzierter Hypoglykämie ausgelöste Somatotropinsekretion vermindert $(18,19,20)$. Nach Garver et al. (21) soll bei unipolar Depressiven und manisch Depressiven eine positive Korrelation zwischen maximaler Somatotropinsekretion im Insulin-Hypoglykämie-Test und Ausscheidung von 3-Methoxy-4-hydroxyphenylglycolaldehyd, dem Hauptmetaboliten des Noradrenalin-Abbaus, bestehen. Weiterhin weist die eingeschränkte Stimulierbarkeit der Somatotropinsekretion mit Amphetamin, das eine Freisetzung von Noradrenalin und Dopamin bewirkt, auf eine Funktionsstörung catecholaminerger Neurone bei endogener Depression hin (22). Im Schlaf und nach Hitzestimulation sind ebenfalls Veränderungen der Somatotropinsekretion gefunden worden (23).

Die regulatorische Wirkung von Lithium auf den Catecholamin-Stoffwechsel zerebraler Neurone wird als ein wesentlicher therapeutischer Effekt bei endogen Depressiven angesehen. Die Wirkung von Lithium auf die Sekretion des Hypophysenvorderlappens ist bisher nur beim Thyrotropin näher untersucht worden. So wird bei einer langdauernden Lithiumtherapie eine gesteigerte Thyrotropinsekretion beobachtet $(24,29)$. Weitgehend unklar ist, ob die Sekretion anderer, catecholaminerg gesteuerter Hormone (Somatotropin, Prolactin, Corticotropin) unter Lithiumbehandlung verändert ist.

In ${ }^{\circ}$ der vorliegenden Arbeit wird die hypophysäre Sekretion von Somatotropin, Corticotropin (über die Cortisolbestimmung), Prolactin und Thyrotropin unter Ruhe- und Stimulationsbedingungen bei depressiv Erkrankten sowie 
Lithium-behandelten Patienten untersucht. Ziel dieser Arbeit ist es, anhand einer größeren Patientenzahl die differentialdiagnostische Wertigkeit des kombinierten hypophysären Stimulationstestes zur Differenzierung depressiver Syndrome zu untersuchen. Die Durchführung des endokrinologischen Funktionstestes während der depressiven Phase und nach abgeklungener Depression soll Veränderungen der klinisch-chemischen Kenngrößen im Krankheitsverlauf und mögliche phasenunabhängige Funktionsstörungen aufzeigen. Außerdem wurde der Einfluß symptomatologischer Kriterien, wie Agitiertheit und Gehemmtheit, auf den Verlauf der hypophysärenSekretion geprüft. Schließlich ermöglichen Untersuchungen der Sekretion hypophysärer Hormone Rückschlüsse auf die Funktion beteiligter zerebraler Neurotransmitter.

\section{Methodik}

Untersucht wurden 48 depressiv erkrankte Frauen, die in Alter und Körpergewicht weitgehend übereinstimmten (Tab. 1). Einflußgrößen wie Diabetes, Hypertonie und Übergewicht, wurden zuvor ausgeschlossen.

Die beiden gleich großen Gruppen der endogen (unipolar, bipolar II, Involutionsdepression) oder reaktiv (in geringerem Maße neurotisch) depressiv erkrankten Frauen stimmen in der mit Selbst- und Fremdbeurteilungsskalen gemessenen Depressionstiefe überein (Beck'scher Depressionsfragebogen, Befindlichkeitsskala und Depressionsskala von $\nu$. Zerssen, Hamiltan-Meßskala).

Die in unserer Untersuchung endogen genannten Depressionen entsprechen weitgehend den primär affektiven Erkrankungen nach Feighner et al. (12) und sind operational eindeutiger defjniert als die reaktiven (neurotischen) Depressionen. Die Phasenzahl war bei den endogen Depressiven $(\bar{x}=4)$ größer als bei den reaktiv Depressiven $(\bar{x}=2)$. Neben der nosologischen Differenzierung des depressiven Syndroms wurde aufgrund des klinischen Urteils eine symptomatologische Unterteilung in eine eher gehemmte oder agitiert-ängstliche Depression vorgenommen. Mindestens 1 Woche vor Durchführung des hypophysären Stimulationstestes wurde eine medikamentöse Behandlung (z. B. mit Thymoleptika) abgesetzt. Die endokrinologische Untersuchung er-

Tab. 1. Gruppen der depressiv erkrankten Frauen und Kontrollen (ICD = International Classification of Diseases).

\begin{tabular}{|c|c|c|c|}
\hline $\begin{array}{l}\text { Anzahl } \\
\text { vor } \\
\text { der } \\
\text { Meno- }\end{array}$ & $\begin{array}{l}\text { nach } \\
\text { der } \\
\text { Meno- }\end{array}$ & $\begin{array}{l}\text { Alter } \\
\text { (Jahre) }\end{array}$ & $\begin{array}{l}\text { Relatives } \\
\text { Körper- } \\
\text { gewicht } \\
\text { (\% des Ideal- } \\
\text { gewichts) }\end{array}$ \\
\hline & & $\overline{\mathbf{x}}(\mathbf{s})$ & $\overline{\mathbf{x}}(\mathrm{s})$ \\
\hline
\end{tabular}

\subsection{Endogene Depression}

(ICD: 296.0; 296.2)

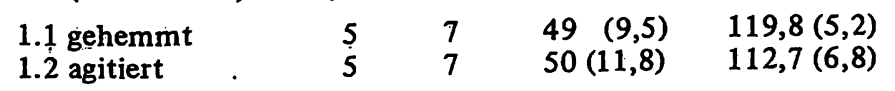

2.0 Reaktive Depression

(ICD: $298.0 ; 300.4$ )

\begin{tabular}{lllll}
2.1 gehemmt & 6 & 6 & $49(10,4)$ & $110,7(5,9)$ \\
2.2 agitiert & 6 & 6 & $46(14,1)$ & $108,3(5,2)$ \\
$\begin{array}{l}\text { 3.0 Lithium-Therapie } \\
\text { (ICD: 296.2; }\end{array}$ & & & & \\
$\begin{array}{l}\text { 296.3) } \\
\text { 4.0 Gesunde }\end{array}$ & 6 & 6 & $45(13,5)$ & $118,4(5,8)$ \\
$\quad$ Kontrollen & 6 & 6 & $48(13,7)$ & $113,4(4,1)$ \\
\hline
\end{tabular}

folgte an 2 Zeitpunkten, und zwar während der Depression (1 Woche nach Aufnahme in die stationäre Behandlung) und nach abgeklungener Depression (am Tag der Entlassung aus stationärer Behandlung). Wesentliche Änderungen des Körpergewich ts zwischen den beiden Zeitpunkten der Untersuchung wurden nicht festgestellt. Eine andere Gruppe früher endogen (unipolar, bipolar II) depressiv Erkrankter (12 Frauen, 4 Männer) wurde vor der Untersuchung 2-7 Jahre (im Mittel 3,9 Jahre) mit Lithium behandelt. Die mittlere Lithiumkonzentration im Serum lag zum Zeitpunkt der Untersuchung bei $0,86 \mathrm{mmol} / 1$. Ein psychosefreies Intervall bestand seit mindestens $2 \mathrm{~J}$ ahren.

Als Kontrolle wurden gesunde Personen (12 Frauen, 5 Männer) jeweils in Anzahl, Geschlecht, Alter und Körpergewicht den Patientengruppen angeglichen, herangezogen. Die Durchführung der Funktionsteste erfolgte am liegenden Probanden in allen Fällen zur gleichen Tageszeit (8.30 Uhr). Zur Stimulation des Hypophysenvorderlappens wurden nach 12-stündiger Nahrungskarenz und unter Ruhebedingungen Thyroliberin $(200 \mu \mathrm{g}$; Relefact, Hoechst) Gonadoliberin ( $50 \mu \mathrm{g}$; GnRH, Serono) und Altinsulin (0,1 I.E./kg Körpergewicht) intravenös gegeben. Kurz zuvor und nach $10,20,30,45,60,90$ und 120 min wurden Blutproben aus einer ständig in einer Armvene liegenden Kanüle für die Bestimmungen von Thyrotropin, Somatotropin, Prolactin, Cortisol (stellvertretend für Corticotropin) und Glucose entnommen. Ausgewertet wurden nur solcne Funktionsteste, bei denen ein Glucoseabfall um mehr als die Hälfte oder unter $2,8 \mathrm{mmol} / 1$ nachweisbar war.

Die Ergebnisse der $L-3,5,3^{\prime}$-Trijod thy ronin-, Thyroxin-, Lutropinund Follitropinbestimmung wurden zur Beurteilung der Schilddrüsen- sowie Ovarienfunktion (Zustand vor oder nach Menopause) herangezogen.

Zur Bestimmung der Hormone wurden mit Ausnahme des Cortisols (Proteinbindungsanalyse) radioimmunologische Methoden (Reagenzien: Somatotropin, Serono; Thyrotropin, Isotopendienst West; Prolactin, Union Carbide; Cortisol, Amersham Buchler) benutzt. Wesentliche Kriterien der methodischen Durchführung und der Qualitätskontrolle zeigt Tabelle 2. Die im Vergleich zum Radioimmunassay geringere Spezifität der Proteinbindungsanalyse des Cortisols kann bei der vorliegenden Fragestellung und Versuchsbedingung vernachlässigt werden. Die methodische Durchführung erfolgte teilmechanisiert mit Hilfe einer Pipettierstation (Micromedic) und Gamma-Probenwechslern (Gamma-Szint-BF 5300, Berthold; Rackgamma, LKB). Die Standardkurven wurden durch Spline-Funktion (Alphatronic-Rechneranlage, Diehl) und Logit-Log-Verfahren approximiert.

Glucose wurde vollmechanisiert (Autoanalyzer II, Technicon) aus Hämolysat nach der Hexokinase/Glucose-6-phosphat-dehydrogenase-Methode (Reagenzien: Boehringer Mannheim) bestimmt (29).

\section{Statistische Auswertung}

Um signifikante Unterschiede (Basalwerte, maximale Stimulationswirkung) zwischen den verschiedenen Probandengruppen zu prüfen, wurden varianzanaly tische Modelle herangezogen (30). So wurde bei den depressiv Erkrankten eine 4-faktorielle Varianzanalyse mit 2 unabhängigen Faktoren (nosologische Gruppen - symptomatologische Gruppen), 2 abhängigen Faktoren (Hormon- und Glucosekonzentrationen - Depressionsstadien) und gleichen Zellfrequenzen, bei Prüfung des Lithiumeinflusses eine 2-faktorielle Varianzanalyse für unabhängige Messungen auf beiden Faktoren (Männer, Frauen - Patienten unter Medikamentenwirkung, gesunde Kontrollen) und ungleichen Zellfrequenzen berechnet.

\section{Ergebnisse}

\section{Depressive Syndrome}

1. Die Basalkonzentrationen der Hormone Somatotropin, Cortisol, Prolactin und Thyrotropin zeigen keine signifikanten Unterschiede zwischen endogen und reaktiv depressiv Erkrankten sowie den gesunden Kontrollen. 
Tab. 2. Methoden der Hormonbestimmungen (Abkürzungen: $B_{0}=$ Aktivität bei der Standardkonzentration $0, T=T o t a l a k t i v i t a ̈ t$, ED $50(85)=$ effektive Dosis bei 50 (85)\%-Bindung, Ak = Antikörper, MRC = Medical Research Council).

\begin{tabular}{|c|c|c|c|c|}
\hline & Somatotropin & Prolactin & Thyrotropin & Cortisol \\
\hline 1. Aufarbeitung der Probe & \multicolumn{4}{|c|}{ Bestimmung direkt aus Serum } \\
\hline 2. Bindendes Protein & $\mathbf{A k}$ & $\mathbf{A k}$ & Ak & Transcortin \\
\hline 3. Markierung des Tracers & ${ }^{125} I$ & ${ }^{125} I$ & ${ }^{125} I$ & ${ }^{75} \mathrm{Se}$ \\
\hline 4. Inkubationszeit bei $20-25^{\circ} \mathrm{C}$ & $\begin{array}{l}\text { 1. Ak: } 24 \mathrm{~h} \\
\text { 2. Ak: } 2 \mathrm{~h}\end{array}$ & $\begin{array}{l}\text { 1. Ak: } 42 \mathrm{~h} \\
\text { 2. Ak: } 5 \mathrm{~h}\end{array}$ & $\begin{array}{l}\text { 1. Ak: } 16 \mathrm{~h} \text { ohne } \\
24 \mathrm{~h} \text { mit } \\
\text { 2. Ak: } 3 \mathrm{~h} \text { "Tracer" }\end{array}$ & $1 / 2 \mathrm{~h}$ \\
\hline 5. „Free-Bound“-Trenntechnik & Doppelantikörper & \multicolumn{2}{|c|}{ Doppelantikörper-Festphase } & $\begin{array}{l}\text { Adsorption } \\
\text { (Sephadex G 25) }\end{array}$ \\
\hline 6. Standard & MRC $66 / 217$ & MRC $71 / 222$ & MRC $68 / 38$ & Cortisol \\
\hline 7. $\dot{B}_{0} / T(\%)(\bar{x} \pm s)$ & $45,9 \pm 3,2$ & $36,8 \pm 2,4$ & $28,0 \pm 3,1$ & \\
\hline 8. $\operatorname{ED~} 50(\mathrm{mU} / \mathrm{l})(\overline{\mathrm{x}} \pm \mathrm{s})$ & $5,8 \pm 0,54$ & $360 \pm 42$ & $7,6 \pm 0,9$ & \\
\hline 9. $\operatorname{ED~} 85(\mathrm{mU} / \mathrm{l})(\overline{\mathrm{x}} \pm \mathrm{s})$ & $1,5.2 \pm 0,17$ & $45 \pm 9$ & $1,5 \pm 0,2$ & \\
\hline $\begin{array}{l}\text { 10. Variationskoeffizient (\%) } \\
\text { von Tag zu Tag, }(\mathbf{x})\end{array}$ & $\begin{array}{l}9,6 \\
(10,5 \mathrm{mU} / \mathrm{l})\end{array}$ & $\begin{array}{l}9,4 \\
(500 \mathrm{mU} / \mathrm{l})\end{array}$ & $\begin{array}{l}10,8 \\
(6,5 \mathrm{mU} / \mathrm{l})\end{array}$ & $\begin{array}{l}7,1 \\
(280 \mathrm{nmol} / \mathrm{l})\end{array}$ \\
\hline
\end{tabular}

2. Vergleicht man die beiden nosologischen Gruppen insgesamt (gehemmte und agitierte Depression zu beiden Untersuchungszeitpunkten), so ergeben sich Unterschie-

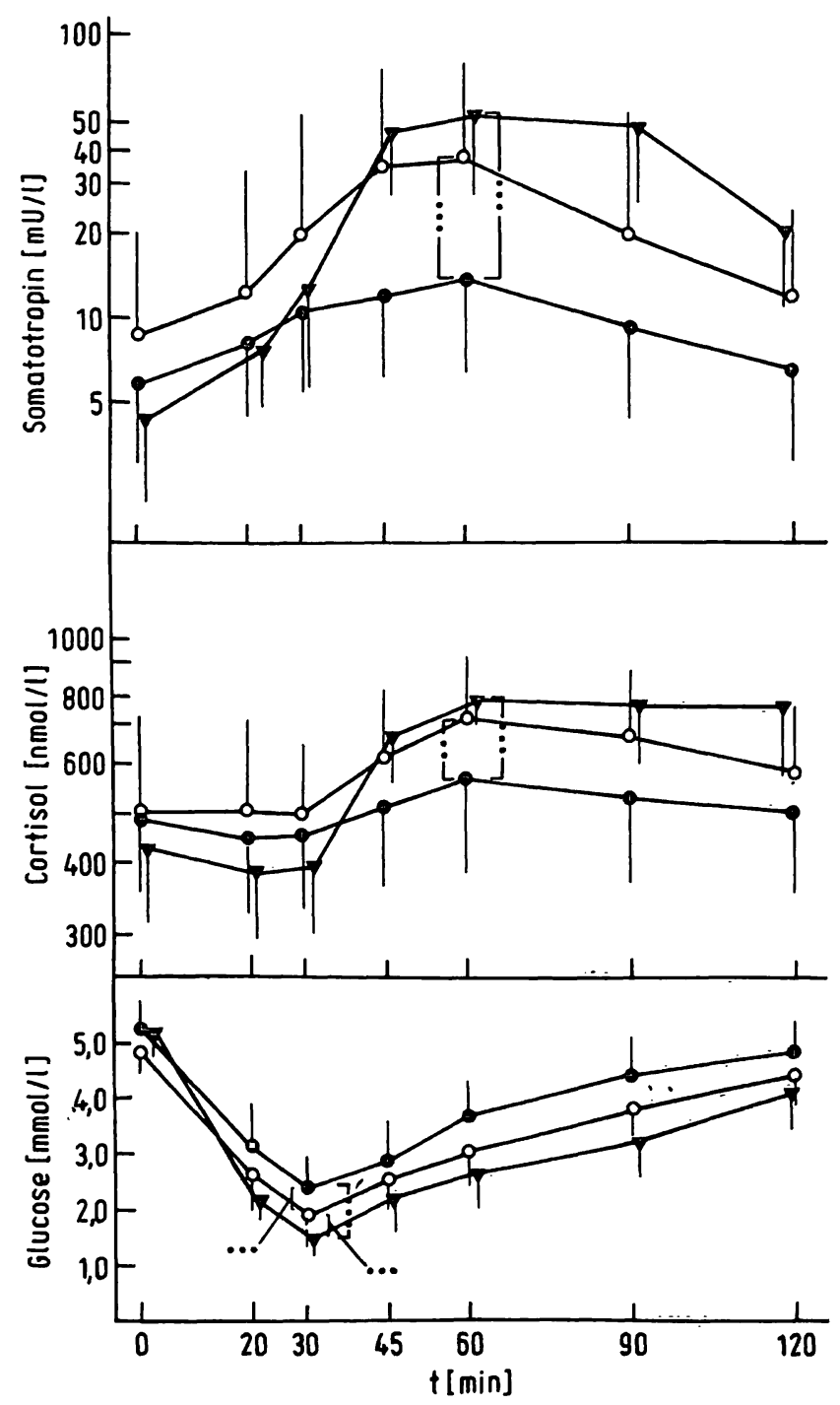

de in der Hormonsekretion nur für Somatotropin und Cortisol. Die Stimulierbarkeit der Somatotropin- und Cortisolsekretion ist bei endogen Depressiven gegenüber reaktiv Depressiven und auch den gesunden Kontrollen deutlich vermindert (Abb. 1, Tab. 3). Auch im Verlauf der Glucosekonzentration unter Insulinbelastung ergeben sich Unterschiede zwischen diesen Gruppen. Bei endogen Depressiven ist der Ausgangswert der Glucosekonzentration höher als bei reaktiv Depressiven, der maximale Glucoseabfall geringer als bei den reaktiv Depressiven und gesunden Kontrollen (Tab. 3).

3. Die korrelationsstatistische Berechnung eines linearen Zusammenhangs zwischen Glucoseminimum und maximaler Somatotropin- bzw. Cortisolantwort ergab für die verschiedenen Probandengruppen sowie während und nach der depressiven Phase Produktmoment-Korrelationskoeffizienten zwischen $\mathrm{r}=-0,03$ und $\mathrm{r}=\mathbf{- 0 , 5 2}$.

4. Wenn man als Grenzwerte für Somatotropin $40 \mathrm{mU} / \mathrm{l}$, Cortisol $690 \mathrm{nmol} / \mathrm{l}$ und Glucose 2,2 mmol/1 zugrunde legt, so sprechen Stimulationsmaxima der Somatotropin- und Cortisolsekretion, die unterhalb des Grenzwertes liegen, und ein maximaler Glucoseabfall, der oberhalb des Grenzwertes liegt, während des depressiven Stadiums der Erkrankung mit einer Vertrạuenswahrscheinlichkeit von 0,9 für eine endogene Depression. In Abbildung 2 sind die 90\%-Bereiche der Somatotropin- und Cortisol-Stimulationsmaxima sowie des maximalen Glucoseabfalls bei endogener und reaktiv/

Abb. 1. Glucoseabfall und Sekretionskinetik von Somatotropin und Cortisol unter hypoglykämischem Stress bei endogen $(\bullet-)$ und reaktiv $(\bullet-0)$ depressiv Erkrankten sowie gesunden Kontrollen ( $\nabla-\nabla)$ : Angegeben sind $\bar{x} \pm s$ (Glucose) und $\log \bar{x} \cdot s^{ \pm 1}$ (Somatotropin, Cortisol) aller Untersuchungen (1. und 2. Zeitpunkt) und das Siğnifikanżniveau $(\bullet \bullet p<0,01$, $\bullet \bullet=p<0,001)$. 


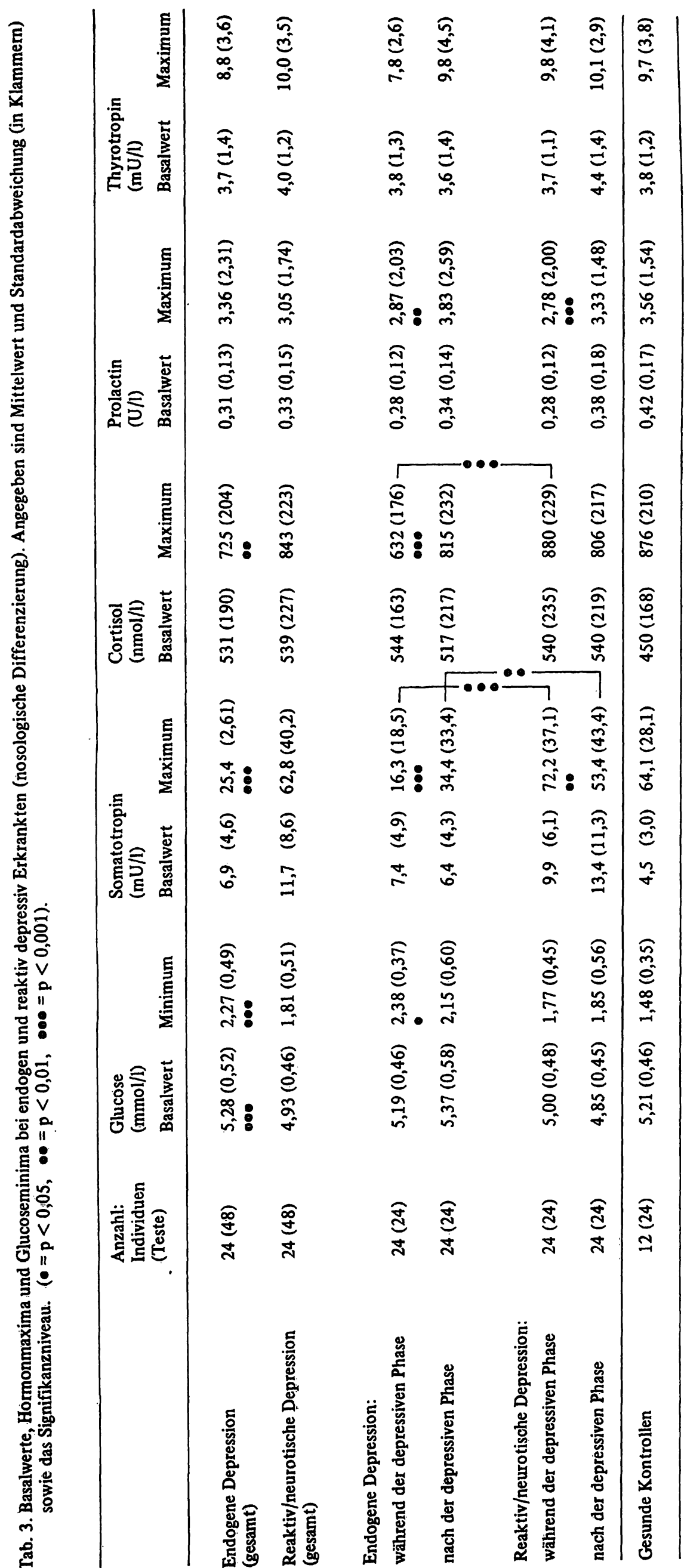




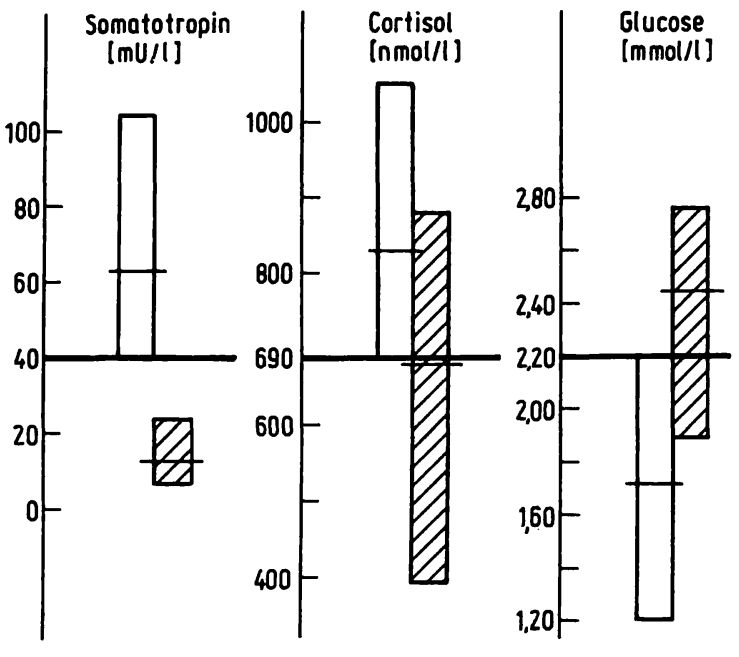

Abb. 2. 90\%-Bereiche und Median der Stimulationsmaxima der Somatotropin- und Cortisolsekretion sowie Glucoseminima bei endogen $(\mathbb{G})$ und reaktiv $(\square)$ depressiv Erkrankten während der depressiven Phase.

neurotischer Depression während der depressiven Phase (1. Zeitpunkt der Untersuchung) aufgeführt.

5. Im Verlaufe der Erkrankung sind bei beiden nosologischen Gruppen Veränderungen in der Stimulierbarkeit der Hormonsekretion und im Glucoseabfall nachweisbar (Tab. 3). Nach abgelaufener endogener Depression (2. Zeitpunkt der Untersuchung) zeigt sich eine Steigerung der Somatotropin- und Cortisolsekretion unter hypoglykämischem Stress (Abb. 3). Auch bei der reaktiv/neurotischen Depression ergeben sich Unterschiede in der Stimulierbarkeit der Somatotropinsekretion zwischen den Depressionsstadien. Hier ist das Stimulationsmaximum der Somatotropinsekretion nach der depressiven Phase niedriger als während der depressiven Phase. Allerdings unterscheiden sich die Stimulationsmaxima der Somatotropinsekretion beider nosologischer Gruppen auch nach klinischer Besserung noch signifikant, während eine Differenz der Cortisol-Stimulationsmaxima zu diesem Zeitpunkt der Untersuchung nicht mehr feststellbar ist.

Obwohl der Glucoseabfall während des Testverlaufs bei endogen Depressiven nach der depressiven Phase gesteigert ist, bleibt er doch im Vergleich mit dem bei reaktiv Depressiven und gesunden Kontrollen weniger stark ausgeprägt. Ein Unterschied im Ausmaß des Glucoseabfalls zwischen den Depressionsstadien ist bei reaktiv Depressiven nicht nachweisbar.

6. Beide nosologischen Gruppen zeigen nach der depressiven Phase eine Zunahme der Stimulierbarkeit der Prolactinsekretion (Tab. 3). Die Differenzierung der depressiv Erkrankten in Gehemmte und Agitierte macht eine unterschiedliche Stimulierbarkeit der Prolactinsekretion dieser symptomatologischen Gruppen sichtbar (Tab. 4). So weisen die Agitierten der reaktiv Depressiven niedrigere Prolactin-Stimula-

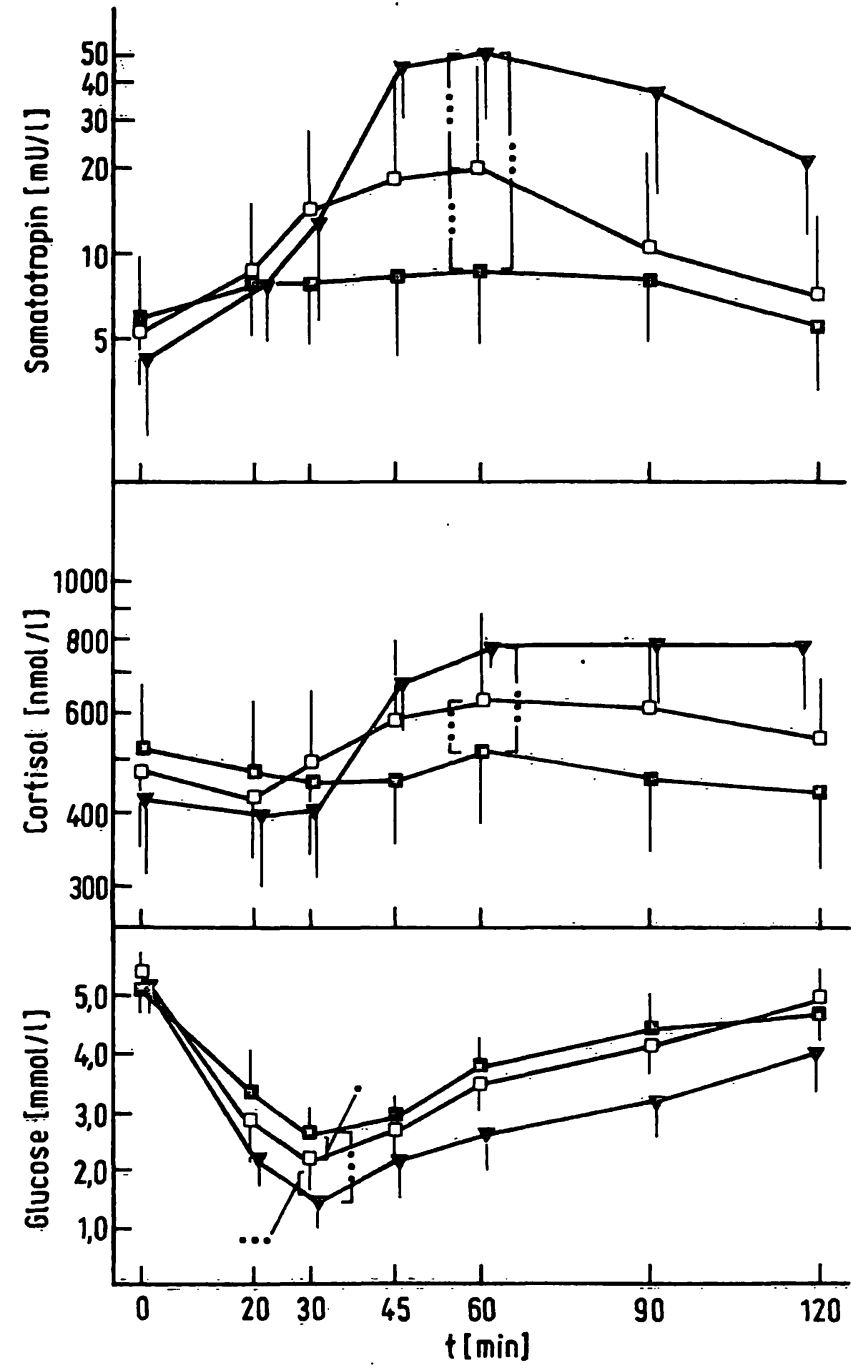

Abb. 3. Glucoseabfall und Sekretionskinetik von Somatotropin und Cortisol unter hypoglykämischem Stress bei endogen depressiv Erkrankten während $(-\square)$ und nach $(\square-0)$ der depressiven Phase sowie gesunden Kontrollen $(\nabla-\nabla)$. Angegeben sind $\bar{x} \pm s$ (Glucose), $\log \bar{x} \cdot s^{ \pm 1}$ (Somatotropin, Cortisol) und das Signifikanzniveau $(\bullet=p<0,05$, $\bullet \bullet=\mathrm{p}<0,001)$.

tionsmaxima auf als die Gehemmten. Nach der depressiven Phase zeigen die Agitierten, nicht dagegen die Gehemmtēn, beider nosologischer Gruppen einen Anstieg der Prolactinsekretion, so daß bei klinischer Besserung kein Unterschied gegenüber den gehemmt Depressiven und gesunden Kontrollen mehr besteht. Weiterhin ergibt sich, daß die basale Glucosekonzentration bei den agitierten Probanden (endogen und reaktiv/neurotische Depressive) höher ist als bei den Gehemmten, während eine Differenz im Ausmaß des Glucoseabfalls nicht feststellbar ist.

7. Unterschiede in der Stimulierbarkeit der Thyrotropinsekretion zwischen den nosologischen und symptomatologischen Gruppen sowie'gesunden Kontrollen ergeben sich nicht (Abb. 3). 
Tab. 4. Basalwerte, Prolactinmaxima und Glucoseminima bei gehemmt und agitiert-ängstlich depressiv Erkrankten (symptomatologi$(\bullet=\mathrm{p}<0,05, \bullet=\mathrm{p}<0,01, \bullet \bullet=\mathrm{p}<0,001)$.

\begin{tabular}{|c|c|c|c|c|c|}
\hline & \multirow{2}{*}{$\begin{array}{l}\text { Anzahl: } \\
\text { Individuen } \\
\text { (Teste) }\end{array}$} & \multicolumn{2}{|l|}{$\begin{array}{l}\text { Glucose } \\
(\mathrm{mmol} / \mathrm{l})\end{array}$} & \multicolumn{2}{|l|}{$\begin{array}{l}\text { Prolactin } \\
\text { (U/I) }\end{array}$} \\
\hline & & Basalwert & Minimalwert & Basalwert & Maximalwert \\
\hline \multicolumn{6}{|l|}{ Endogene Depression: } \\
\hline gehemmt & $12(24)$ & $5,15(0,40)$ & $2,24(0,46)$ & $0,31 \quad(0,12)$ & $3,60(2,46)$ \\
\hline agitiert & $12(24)$ & $5,41(0,64)$ & $2,31(0,58)$ & $0,31 \quad(0,14)$ & $3,11 \quad(2,16)$ \\
\hline \multicolumn{6}{|l|}{ Reaktiv/neurotische Depression: } \\
\hline gehemmt & $12(24)$ & $4,59(0,56)$ & $1,79(0,38)$ & $0,35(0,14)$ & $3,70(1,81)$ \\
\hline agitiert & $12(24)$ & $5,28(0,35)$ & $1,83(0,64)$ & $0,31(0,16)$ & $2,40(1,67)$ \\
\hline \multicolumn{6}{|l|}{ Gehemmte Depression (gesamt): } \\
\hline während der depressiven Phase & $24(24)$ & $4,84(0,42)$ & $2,09(0,44)$ & $0,29(0,10)$ & $3,52(2,03)$ \\
\hline nach der depressiven Phase & $24(24)$ & $4,90(0,54)$ & $1,94(0,40)$ & $0,37(0,16)$ & $3,79(2,23)$ \\
\hline \multicolumn{6}{|l|}{ Agitierte Depression (gesamt): } \\
\hline während der depressiven Phase & $24(24)$ & $5,36(0,52)$ & $2,07(0,38)$ & $0,25(0,14)$ & $2,15(1,99)$ \\
\hline nach der depressiven Phase & $24(24)$ & $5,34(0,49)$ & $2,07(0,76)$ & $0,36(0,16)$ & $3,36(1,84)$ \\
\hline
\end{tabular}

\section{Lithium-behandelte, psychosefreie Probanden}

1. Die Stimulierbarkeit der Somatotropin- und Thyrotropinsekretion bei endogen depressiv erkrankten Frauen, die sich seit mindestens 2 Jahren im psychosefreien Intervall befinden und unter Wirkung einer Lithium-Langzeittherapie stehen, ist in Abbildung 4 dargestellt. Die Stimulationskurve der Somatotropinsekretion ist bei Lithium-behandelten Probanden gegenüber nicht-behandelten endogen Depressiven deutlich angehoben und zeigt einen Verlauf wie bei gesunden Kontrollen.

Unter Lithiumtherapie wird im Vergleich mit nichtbehandelten endogen Depressiven und gesunden Kontrollen ein starker Anstieg der Thyrotropinsekretion (Basal- und Stimulationswerte) gefunden. Eine Wirkung auf die Konzentration von Trijodthyronin und Thyroxin im Serum ist nicht nachweisbar.

2. Eine Unterteilung der Lithium-behandelten Probanden in Frauen vor und nach der Menopause sowie Männer läßt eine unterschiedliche Stimulierbarkeit der Somatotropin-, Cortisol- und Thyrotropinsekretion dieser Gruppen erkennen (Tab. 5). Lithiumbehandelte Frauen vor der Menopause und Männer weisen höhere Stimulationsmaxima der Somatotropinsekretion auf als gesunde Kontrollen, während bei Frauen nach der Menopause die Stimulationsmaxima niedriger sind als bei gesunden Kontrollen.

Ebenso liegen die Stimulationsmaxima der Cortisolsekretion der Lithium-behandelten Frauen nach der Menopause tiefer als die der gesunden Kontrollen.
Die Cortisol-Stimulationsmaxima der Frauen vor der Menopause und Männer dagegen unterscheiden sich unter langdauernder Lithium-Therapie nicht mehr signifikant von denen gesunder Kontrollen.

Die Stimulationsmaxima der Thyrotropinsekretion zeigen bei Frauen nach der Menopause im Gegensatz zu Frauen vor der Menopause keinen signifikanten Anstieg gegenüber gesunden Kontrollen.

3. Ein Lithiumeffekt auf die Stimulierbarkeit der Prolactinsekretion läßt sich nicht nachweisen.

\section{Diskussion}

Unsere Untersuchungen zeigen, daß die nosologische Differenzierung der depressiven Syndrome in eine endogene und reaktiv (neurotische) Form mit Hilfe des Insulin-Hypoglykämie-Testes (Bestimmung von Somàtotropin, Cortisol und Glucose) möglich ist. Besonders die eingeschränkte Stimulierbarkeit der Somatotropinsekretion bei endogen Depressiven während der depressiven Phase erlaubt eine klare Abgrenzung von reaktiv (neurotisch) Depressiven (Abb. 2). Höchstwerte der Somatotropinkonzentration im Serum unterhalb von $40 \mathrm{mU} / 1$ sprechen mit einer Vertrauenswahrscheinlichkeit von 0,9 für eine endogene Depression. Bei nur einer untersuchten Person mit endogener Depression wurde ein Stimulationsmaximum oberhalb dieses Wertes gefunden.

Bisherige Ergebnisse weisen darauf hin, daß die gesteigerte Sekretion nach Insulin-induzierter Hypoglykämie vorwiegend durch noradrenerge Neurone vermittelt wird 
Tab. 5. Basalwerte und Hormonmaxima bei Lithium-behandelten Probanden (mindestens $2 \mathrm{~J}$ ahre psychosefreies Intervall). Angegeben sind Mittelivert und Standardabweichung (in Klammern) sowie das Signifikanzniveau

$(\bullet=\mathrm{p}<0,05, \bullet=\mathrm{p}<0,01, \cdots=\mathrm{p}<0,001)$.

\begin{tabular}{|c|c|c|c|c|c|c|c|}
\hline & \multirow[t]{2}{*}{$\mathbf{N}$} & \multicolumn{2}{|c|}{$\begin{array}{l}\text { Thyrotropin } \\
(\mathrm{mU} / \mathrm{l})\end{array}$} & \multicolumn{2}{|c|}{$\begin{array}{l}\text { Somatotropin } \\
(\mathrm{mU} / \mathrm{l})\end{array}$} & \multicolumn{2}{|l|}{$\begin{array}{l}\text { Cortisol } \\
(\mathrm{nmol} / \mathrm{l})\end{array}$} \\
\hline & & Basalwert & Maximum & Basalwert & Maximum & Basalwert & Maximum \\
\hline \multicolumn{8}{|c|}{ Frauen vor der Menopause: } \\
\hline Lithiumtherapie & 5 & $14,0(12,0)$ & 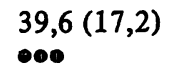 & $13,0(12,0)$ & $118 \quad(62,0)$ & $406(69)$ & 739 (108) \\
\hline Gesunde Kontrollen & 5 & $3,2 \quad(1,2)$ & $10,8 \quad(5,2)$ & $7,2 \quad(9,8)$ & $74,4(35,4)$ & $397(105)$ & $792(63)$ \\
\hline \multicolumn{8}{|c|}{ Frauen nach der Menopause: } \\
\hline Lithium therapie & 6 & $5,6 \quad(2,0)$ & $16,0(12,4)$ & $8,2 \quad(3,4)$ & $35,8(10,6)$ & $475(234)$ & $665(110)$ \\
\hline Gesunde Kontrollen & 6 & $3,6 \quad(0,4)$ & $7,2 \quad(2,0)$ & $3,0 \quad(1,4)$ & $55,4(24,0)$ & $493(217)$ & $1040(234)$ \\
\hline Männer: & & . & & & & & \\
\hline Lithiumtherapie & 4 & $4,4 \quad(1,6)$ & $12,4 \quad(2,0)$ & $3,8 \quad(4,0)$ & $113 \quad(31,0)$ & $530(154)$ & $712(132)$ \\
\hline Gesunde Kontrollen & 5 & $2,4 \quad(0,4)$ & $6,4 \quad(1,2)$ & $3,2 \quad(0,8)$ & $65,6(32,4)$ & $524(137)$ & 737 (193) \\
\hline
\end{tabular}

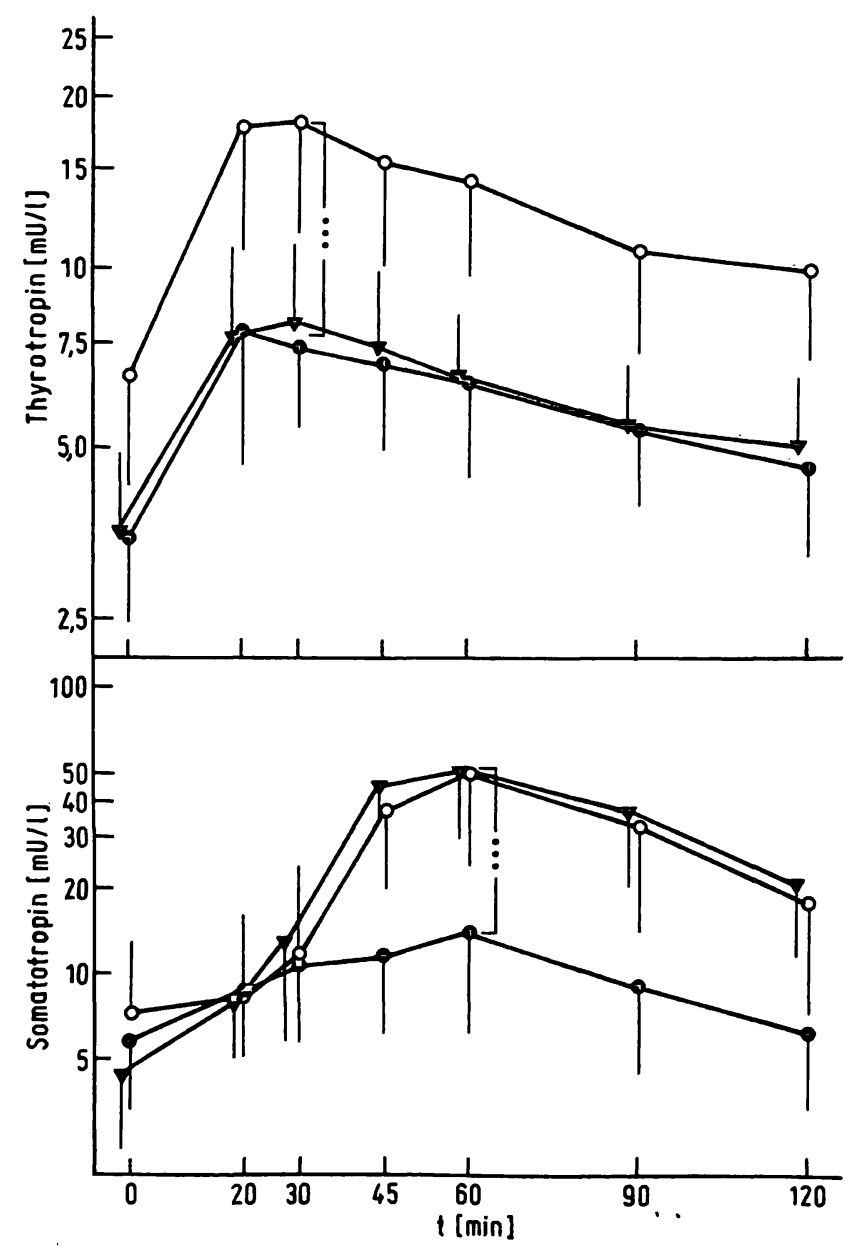

Abb. 4. Sekretionskinetik von Thyrotropin und Somatotropin nach Thyroliberin- und Insulin-Applikation bei endogen depressiv Erkrankten $(\bullet \bullet)$, Lithium-behandelten, psychosefreien Probanden $(0-0)$ und gesunden Kontrollen $(\nabla \longrightarrow \nabla)$.

Angegeben sind $\log \overline{\mathrm{x}} \cdot \mathrm{s}^{ \pm 1}$ und das Signifikanzniveau $(\bullet \bullet \bullet=\mathrm{p}<0,001)$.
$(21,31)$. Die abgeschwächte Somatotropinantwort bei endogener Depression unterstützt die Hypothese einer reduzierten, funktionalen noradrenergen Aktivität.

Es ist unklar, ob der nur geringe Anstieg der Somatotropinsekretion bei endogener Depression überhaupt auf die stressorische Wirkung der Hypoglykämie zurückzuführen ist, oder in einem inapprobiaten Effekt des Thyroliberins seine Ursache hat. Während die Thyroliberingabe bei Gesunden keinen Anstieg der Somatotropinsekretion bewirkt, wird bei einigen Erkrankungen, wie z. B. der Akromegalie und primären Hypothyreose, eine erweiterte Rezeptorsensibilität der Somatotropin-produzierenden Zellen im Hypophysenvorderlappen für Thyroliberin beobachtet $(32,33)$.

Auch bei endogen Depressiven wurde ein Thyroliberininduzierter Sekretionsanstieg des Somatotropins beschrieben (34).

Versuche, ein Catecholamindefizit nachzuweisen, haben bisher nicht zu eindeutigen Ergebnissen geführt (35). Eine verminderte Ausscheidung von 3-Methoxy-4hydroxyphenylglycolaldehyd im Urin bei deprèssiv Erkrankten ist von mehreren Autoren beschrieben worden $(36,37)$. Allerdings ist umstritten, in welchem Ausmaß das im Urin ausgeschiedene 3-Methoxy-4hydroxyphenylglycolaldehyd aus dem Gehirn stammt, und nicht auch die Aktivität des peripheren sympatischen Nervensystems widerspiegelt (38).

Die Untersuchung der 3-Methoxy-4-hydroxyphenylglycolaldehyd-Konzentration im Liquor bei endogen De̊pressiven führte zu unterschiedlichen Ergebnissen. Es wurden im Vergleich mit gesunden Kontrollen erniedrigte (39) und unveränderte Konzentrationen (40) gefunden. Im autoptischen Hirngewebe konnte keine Er- 
niedrigung von Noradrenalin festgestellt werden. Die berichtete Dopaminverminderung im Nucleus ruber (41) wurde von anderen Autoren (42) nicht bestätigt. $\mathrm{Da}$ es bisher nicht eindeutig gelungen ist, die Funktionsstörung aminerger Neurone bei endogener Depression auf ein Amindefizit zurückzuführen, sind in neuerer Zeit hypothetische Überlegungen über eine herabgesetzte Empfindlichkeit postsynaptischer Aminrezeptoren in den Vordergrund gerückt. Neuroendokrinologische Untersuchungen mit Apomorphin, einem Dopaminrezeptorstimulator, haben eine normale Somatotropinsekretion bei endogen Depressiven ergeben (20), so daß die Funktion von Dopaminrezeptoren nicht verändert erscheint. Mit Clonidin dagegen, einem $\alpha$-adrenergen Rezeptorstimulator, wurde bei endogen Depressiven eine geringere Stimulierbarkeit der Somatotropinsekretion festgestellt als bei reaktiv, neurotisch Depressiven (43).

Vor allem die Ergebnisse der Stimulationsversuche mit Clonidin führten Matussek (43) dazu, zwischen praeund postsynaptischen Depressionen zu unterscheiden, die den schon früher von Ashcroft et al. (44) angenommenen "lower-sensitivity" und "low out-put depression" entsprechen. Nach dieser Hypothese sind reaktive, neurotische Depressionen und Erschöpfungsdepressionen durch einen praesynaptischen Aminmangel bei normaler oder kompensatorisch gesteigerter postsynaptischer $\mathrm{Re}$ zeptorempfindlichkeit, endogene Depressionen durch eine herabgesetzte postsynaptische Rezeptorempfindlichkeit gekennzeichnet.

In unseren Untersuchungen bei depressiv Erkrankten während der depressiven Phase zeigt sich, daß Höchstwerte der Cortisolkonzentration im Serum nach Insulininduzierter Hypoglykämie unterhalb $690 \mathrm{nmol} / 1 \mathrm{mit}$ einer Vertrauenswahrscheinlichkeit von 0,9 für eine endogene Depression sprechen. Bei $33 \%$ der endogen Depressiven lag das Stimulationsmaximum der Cortisolsekretion oberhalb dieses Wertes, so daß in diesen Fällen eine klare Abgrenzung von reaktiv (neurotisch) Depressiven durch Beurteilung der Cortisolsekretion allein nicht möglich ist.

Auch mit Hilfe des Dexamethason-Hemmtestes lassen sich endogen und neurotisch Depressive unterscheiden, allerdings weniger präzise als im Insulin-HypoglykämieTest. Carroll et al. (10) fanden bei ,endogenomorphen“ Depressiven in $62 \%$ der Fälle eine geringere Suppression der Cortisolsekretion als bei neurotisch Depressiven und gesunden Kontrollen. Im Vergleich mit dem Dexamethason-Hemmtest erlaubt der Insulin-Hypoglykämie-Test in einem höheren Prożentsatz eine Differenzierung depressiver Syndrome, da neben der Cortisolsekretion auch die Somatotropinsekretion und der Glucoseabfall zur Beurteilung herangezogen werden können.

Auch im Ausmaß des Glucoseabfalls ergeben sich Unterschiede, die zur Differentialdiagnose depressiver Syndrome beitragen. Nach unseren Untersuchungen weisen Glucoseminima oberhalb von $2,2 \mathrm{mmol} / 1$ während der depressiven
Phase mit einer Vertrauenswahrscheinlichkeit von 0,9 auf eine endogene Depression hin. Schon von Mueller et al. (18) wurde beschrieben, daß endogen Depressive nach intravenöser Applikation von Insulin einen geringeren Glucoseabfall zeigen, als neurotisch Depressive. Die Autoren fanden während der depressiven Phase, nicht dagegen nach abgeklungener Erkrankung, eine Korrelation zwischen Ausmaß des Glucoseabfalls und Stärke der Somatotropinantwort. In unseren Untersuchungen ergibt sich bei allen Versuchsgruppen sowie während und nach der depressiven Phase keine lineare Abhängigkeit zwischen Grad des Glucoseabfalls und maximaler Somatotropin- bzw. Cortisolantwort (ein Glucoseabfall um mehr als die Hälfte oder unter $2,8 \mathrm{mmol} / \mathrm{l}$ vorausgesetzt), so daß die eingeschränkte Stimulierbarkeit der Somatotropin- und Cortisolsekretion bei endogen Depressiven nicht durch einen verminderten Glucoseabfall erklärt wird.

Die Ursache des geringeren Insulin-induzierten Glucoseabfalls bei endogen Depressiven ist bisher unklar. $\mathrm{Ob}$ eine verstärkte hormonelle Gegenregulation oder eine gestörte zelluläre Glucoseaufnahme bei dieser Erkrankung in Betracht kommen, müssen weitere biochemische Untersuchungen zeigen.

Nach abgeklungener endogener Depression (2. Zeitpunkt der Untersuchung) ist eine Steigerung der Somatotropinund Cortisol-Stimulierbarkeit sowie des Glucoseabfalls nachweisbar (Abb. 3). Allerdings ergeben sich auch zu diesem Zeitpunkt der Untersuchung noch deutliche Unterschiede im Vergleich mit gesunden Kontrollen. So liegen bei endogen Depressiven 58\% der SomatotropinStimulationsmaxima unterhalb, $75 \%$ der Glucoseminima oberhalb des Bereiches gesunder Kontrollen. Endogen Depressive, die sich seit mindestens $2 \mathrm{Jahren}$ im psychosefreien Intervall befinden und prophylaktisch mit Lithium behandelt werden, zeigen in keinem Fall Stimulationsmaxima der Somatotropin- und Cortisolsekretion außerhalb des Bereiches der gesunden Kontrollen, so daß anzunehmen ist, daß bei einem Teil der endogen Depressiven zum 2. Zeitpunkt der Untersuchung die depressive Phase noch nicht vollständig abgeklungen ist. Die Tatsache aber, daß bei den Lithium-behandelten psychosefreien Probanden noch in einem höheren Prozentsatz (66\%) ein geringerer Glucoseabfall gefunden wird als bei gesunden Kontrollen, deutet darauf hin, daß diesem phasenunabhängigen Defekt ein tiefgreifender pathobiochemischer Mechanismus bei endogener Depression zugrunde liegt.

Die Stimulationsmaxima der Thyrotropinsekretion weisen in unseren Untersuchungen keine Unterschiede zwischen den nosologischen Gruppen und gesunden Kontrollen auf. Andere Ergebnisse werden von mehreren Autoren berichtet, die bei endogen depressiv $\mathrm{Er}$ krankten gehäuft eine abgeschwächte Stimulierbarkeit der Thyrotropinsekretion nach alleiniger Thyroliberingabe fanden $(1,45,46,47,48)$. Die fehlende Ubereinstimmung in der Testdurchfuihrung, vor allem in der 
Thyroliberin-Dosierung und in den Kriterien der Bewertung einer nicht ausreichenden Stimulation erschweren einen Vergleich der Ergebnisse. So ist bei unseren Untersuchungen ein möglicher Effekt des gleichzeitig bestehenden hypoglykämischen Stress auf die Thyrotropinsekretion in Betracht zu ziehen.

Die Stimulierbarkeit der Prolactinsekretion durch Thyroliberin wird durch das Symptom Agitiertheit beeinflußt. Bei den Agitierten beider nosologischen Gruppen ist nach Besserung der Erkrankung eine Steigerung der Prolactin-Stimulierbarkeit nachweisbar. Der Angriffspunkt der Thyroliberin-Wirkung liegt auf hypophysärer Ebene. Möglicherweise führen Angst und Agitiertheit während der depressiven Phase über eine gesteigerte inhibitorische Aktivität dopaminerger Neurone zu einer verminderten Stimulation der Prolactinsekretion durch Thyroliberin.

Linnoila (49) beschreibt eine eingeschränkte ProlactinStimulierbarkeit nach Thyroliberin-Gabe bei 11 von 12 unipolar depressiv Erkrankten, die allerdings nicht symptomatologisch differenziert wurden.

Eine verminderte Stimulierbarkeit der SomatotropinSekretion ist bei endogen depressiv Erkrankten, die im psychosefreien Intervall langdauernd mit Lithium behandelt werden, nicht mehr nachweisbar. Bei den Lithiumbehandelten Frauen vor der Menopause und Männern ist die Somatotropin-Stimulierbarkeit sogar im Vergleich mit gesunden Kontrollen gesteigert.

Ein zusammenhängendes Bild über die Lithiumwirkung auf den Neurotransmitterstoffwechsel besteht bisher noch nicht, obwohl unterschiedliche Einzeleffekte beschrieben wurden (50). Die bisherigen Ergebnisse weisen darauf hin, daß Lithium cAMP-vermittelte intrazelluläre Prozesse, die durch Peptidhormone ausgelöst werden, besonders in Niere, Schilddrüse und im Zentralnervensystem beeinflußt (51). So wird der häufig zu beobachtende nephrogene Diabetes insipidus zum Teil auf eine verminderte Vasopressin-induzierte cAMP-Bildung unter Lithiumwirkung zurückgeführt $(52,53)$. Es liegt nahe, ebenso die Störung der Schilddrüsenfunktion, die auch in unseren Untersuchungen Veränderungen im Sinne einer primären Hypothyreose (erhöhte Thyrotropin-Basalsekretion und gesteigerte Stimulierbarkeit der Thyrotropinsekretion) zeigt, durch eine inhibitorische Wirkung des Lithiums auf die Adenylatcyclase-Aktivität in der Schilddrüsenzelle zu erklären. Außerdem scheinen Prozesse nach der cAMP-Bildung, wie z. B. die calciumabhängige Freisetzung von Thyroxin, unter langdauernder Lithiumwirkung eingeschränkt zu sein (54). Veränderungen der peripheren
Triiodthyronin- und Thyroxin-Konzentrationen wurden allerdings von uns nicht festgestellt.

Innerhalb der Gruppe der Lithium-behandelten, psychosefreien Probanden zeigen die Frauen nach der Menopause abweichende Ergebnisse im Stimulationstest. Im Vergleich mit gleichaltrigen, gesunden Kontrollen (post Menopause) ist eine signifikante Zunahme der Thyrotropin-Stimulierbarkeit nicht nachweisbar. Die Stimulationsmaxima der Somatotropinsekretion liegen im Mittel unterhalb von denen gesunder Kontrollen. Auch die Cortisol-Stimulierbarkeit ist signifikant geringer, während sich bei Lithiumbehandelten Männern und Frauen vor der Menopause kein Unterschied der Cortisol-Stimulierbarkeit gegenüber gesunden Kontrollen ergibt. Trotz Psychosefreiheit und Lithiumprophylaxe sind also bei den Frauen nach der Menopause neuroendokrine Reaktionen feststellbar, die Merkmale der endogenen Depression daršrtellen.

Stimulationsversuche mit Desimipramin (55), das durch Hemmung des präsynaptischen Rücktransportes vor allem von Noradrenalin zu einem Somatotropinanstieg führt, und Clonidin (43) haben ergeben, daß auch gesunde Frauen nach der Menopause keinen Unterschied der Stimulierbarkeit der Somatotropinsekretion gegenüber endogen depressiv Erkrankten zeigen. Neben einem Catecholaminmangel und/oder einer geringeren $\alpha$-Rezeptorempfindlichkeit zentraler Neurone bei endogener Depression sind daher zusätzliche unbekannte Funktionsstörungen in Betracht zu ziehen.

Die pathobiochemischen Ursachen für das Auftreten depressiver Syndrome sind weitgehend unklar. Die nosologische und symptomatologische Differenzierung der Erkrankung stützt sich bisher wesentlich auf subjektive Kriterien, wie klinisches Urteil und Angaben des Patienten. Unsere Untersuchungen zeigen, daß es möglich ist, die gestörte Funktion zentralnervöser Systeme bei depressiv Erkrankten über Veränderungen der neuroendokrinen Sekretion zu erfassen und differentialdiagnostisch zu nutzen. Der hypophysäre Stimulationstest trägt somit dazu bei, die Klassifizierung depressiver Syndrome auf eine objektivere Basis zu stellen.

\section{Danksagung}

Wir bedanken uns bei Herrn Dr. E. M. Steinmeyer, Abteilung Psychiatrie der Rhein.-Westf. Technischen Hochschule Aachen, für die statistische Beratung.

Frau B. Witte danken wir für die sorgfältige experimentelle Mitarbeit.

\section{Literatur}

1. Ettigi, P. G. \& Brown, G. M. (1977), Am. J. Psychiatry 134, 493-501.

2. Schildkraut, J. J. (1975), in: American Handbook of Psychiatry, (Hamburg, D. A. \& Brodie, H. K. H. ed.) Inc.

Publishers, New York, Vol. 6, S. 460-487.

3. Goodwin, F. K. \& Post, R. M. (1975), in Biology of the Major Psychoses, (Freedmann, D. W., ed.) Raven Press, New York, S. 299-332. 
4. Van Loon, G. R. (1973), in: Frontiers in Neuroendocrinology (Ganong, F. W. \& Martini, L., ed.) Oxford University Press, New York, S. 209-247.

5. Frohman, L. A. \& Stachura, M. E. (1975), Metabolism 24, 211-234.

6. Sachar, E. J., Roffwarg, H. P., Gruen, P. H., Altmann, N. \& Sassin, J. (1976), Pharmakopsych. 9, 11-17.

7. Carroll, B. J., Curtis, G., Mendels, J. \& Sugermann, A. (1976), Arch. Gen. Psychiatry 22, 1039-1958.

8. Doerr, P., v. Zeerssen, D. \& Schulz, H. (1979), diese Z., 17, 147.

9. Klein, D. F. (1974), Arch. Gen. Psychiatry 31, 447-454.

10. Caroll, B. J. (1978), in: Depressive Disorders, Symposium Rome, May 1977, (Garattini, S., ed.) Schattauer Verlag, Stuttgart-New York, S. 233-236.

11. Caroll, B. J., Greden, J. F., Rubin, R. T., Hasekett, R., Feinsberg, M. \& Steingart, D. (1978), Acta Endocrinol 89, Supp. 220, 14.

12. Schlesser, M. A., Winokur, G. \& Sherman, B. M. (1979), Lancet, $I, 739-741$.

13. Perez-Reyes, M. (1972), in: Recent advances in the psychobiology of depressive illness, Goverment printing office, Washingtrn, S. 131-135.

14. Martin, J. B. (1973), N. Engl. J. Med 288, 1384-1393.

15. Müller, E. E. (1973), Neuroendocrinology, 11, 339-369.

16. Leebaw, W. F., Lee, L. A. \& Woolf, P. D. (1978), J. Clin. Endocrinol. Metab. 47, 480-487.

17. Bivens, C. H., Lebovitz, H. E. \& Felman, J. M. (1973), N. Engl. J. Med., 289, 236-239.

18. Mueller, P. S., Henninger, G. R. \& McDonald, P. K. (1969), Arch. Gen. Psychiatry 21, 587-594.

19. Endo, M., Endo, J., Nishikubo, M., Yamaguchi, T. \& Hatotani, N. (1974), in: Psychoendocrinology, Workshop Conf. Int. Soc. Psychoendocrinology, Mieken 1973, Verlag Karger, Basel, S. 22-31.

20. Caspar, R. C., Davis, J. M., Pandey, G. N. \& Garver, D. L. (1977), Psychoneuroendocrinology 2, 105-114.

21. Garver, D. L., Pandey, G. N., Dekirmenjian, H. \& DeleonJones, F. (1975), Am. J. Psychiatry 132, 1149-1154.

22. Langer, G., Heinze, G., Reim, B. \& Matussek, N. (1976), Arch. Gen. Psychiatry 33, 1471-1475.

23. Schilkrut, R., Chandra, O., Osswald, M., Ruther, E., Baarfüsser, B. \& Matussek, N. (1975), Neuropsychobiology 1, 70-79.

24. Lazarus, J. H. \& Bennie, E. H. (1972), Acta Endocrinol. 70, 266-272

25. Emerson, C. H., Dyson, W. L. \& Utiger, R. D. (1973), J, Clin. Endocrinol. Metab. 36, 338-346.

26. Lauridsen, U. B., Kirkegaard, C. \& Nerup, J. (1974), J. Clin. Endocrinol. Metab. 39, 383-385.

27. Takahashi, S., Kondo, H., Yoshimura, M. \& Ochi, Y. (1975), Folia Psychiatr. Neurol. Jpn. 29, 231-237.

28. Feighner, J. P., Robins, E., Guze, S. B., Woodruff, R. A., Winokur, G. \& Munoz, R. (1972), Arch. Gen. Psychiatry 26, $57-63$.
29. Bergmeyer, H. U., Bernt, E., Schmidt, F. \& Storck, H. (1974), in: Methoden der enzymatischen Analyse, (Bergmeyer, H. U., ed.) Verlag Chemie, Weinheim, S. 1241-1246.

30. Kirk, R. E. (1968), Experimental Design: Procedures for the Behavioral Sciences, Brooks/Cole Publishing Comp. Belmont, California.

31. Lal, S., Tolis, G., Martin, J. B., Brown, G. M. \& Guyda, H. (1975), J. Clin. Endocrinol. Metab. 41, 827-832.

32. von Werder, K., Fahlbusch, R., Gay, R., Pickardt, C. R. \& Schultz, B. (1976), Klin. Wochenschr. 54, 335-338.

33. Hamada, N., Noi, K., Nishizawa, Y., Okamoto, T., Hasegawa, K., Morii, H. \& Wada, M. (1976), Endocrinol. Jpn. 23, 5-10.

34. Brambilla, F., Smeraldi, E., Sacchetti, E., Negri, F., Cocchi, D. \& Müller, E. E. (1978), Arch. Gen. Psychiatry 35, 12311238.

35. Beckmann, H. (1978), Nervenarzt 49, 557-568.

36. Jones, F. D., Maas, J. W., Dekirmenjian, H. \& Fawcett, J. A. (1973), Science 170, 300-302.

37. Maas, W., Dekirmenjian, D. \& Fawcett, J. A. (1974), Int. Pharmacopsychiatry 9, 14-26.

38. Maas, J. W., Dekirmenjian, H., Garver, D., Redmond, D. E. \& Landis, D. H. (1973), Eur. J. Pharmacol. 23, 121-130.

39. Post, M., Gordon, K., Goodwin, F. K. \& Bunney, Jr., W. E. (1973), Science 179, 1002-1003.

40. Shaw, D. M., O'Keefe, R., McSweeney, D. A., Brooksbank, B. W. L., Noquera, R. \& Coppen, A. (1973), Psychol. Med. 3, 333-336.

41. Birkmayer. W. \& Riederer, P. (1975), J. Neural Transm. 37, 95-109.

42. Beskow, J., Gottfries, C. G., Roos, B. E. \& Windslad, B. (1976), Acta Psychiatr. Scand. 53, 7-20.

43. Matussek, N. (1978), Nervenarzt 49, 569-575.

44. Ashcroft, G. W., Ecclestone, D., Murray, L. G., Glen, A. J., Crawford, T. B. B., Pullar, J. A., Shields, P. J., Walter, D. S., Blackburn, J. M., Chonnechan, J. \& Lonergan, M. (1972), Lancet II, 573-577.

45. Hollister, L. E., Davis, K. L. \& Berger, P. A. (1976), Arch. Gen. Psychiatry 33, 1393-1396.

46. Gold, P. W., Goodwin, F. K., Wehr, T. \& Rebar, R. (1977), Am. J. Psychiatry $134,1028-1031$.

47. Gregoire, F., Braumann, H., De Buck, R. \& Corvilain, J. (1977), Psychoendocrinology 2/3, 303-312.

48. Loosen, P. T., Prange, A. J. \& Eilson, I. C. (1978), Am. J. Psychiatry 135, 244-246.

49. Linnoila, M. (1978), Acta Endocrinol. 89, Suppl. 220, 15.

50. Bunney, W. E. \& Murphy, D. L. (1976), Pharmacopsych. 9 , $142-147$.

51. Singer, I. \& Rotenberg, D. (1973), N. Engl. J. Med. 289, $254-260$.

52. Dousa, T. P. \& Hechter, O. (1970), Life Sci. 9, 765.

53. Singer, I., Totenberg, D. \& Puschett, J. (1972), J. Clin. Invest. $51,1081-1091$.

54. Williams, J. A., Berens, S. C. \& Wolff, J. (1971), Endocrinology $88,1385-1388$.

55. Laakmann, G. (1980), Nervenarzt 51, im Druck.
Dr. med. Knut Kleesiek Abteilung Klinische Chemie und Pathobiochemie der Rhein.-Westf. Technischen Hochschule Aachen Goethestr. 27-29 D-5100 Aachen

Dr. med. Arnold Eberhard Brauhausstraße 4 D-4600 Dortmund 1 
